\title{
Caracterización de pacientes con cirrosis hepática y bacteriemia de un hospital universitario en Medellín, Colombia
}

\section{Characterization of patients with liver cirrhosis and bacteremia treated at a university hospital in Medellín-Colombia}

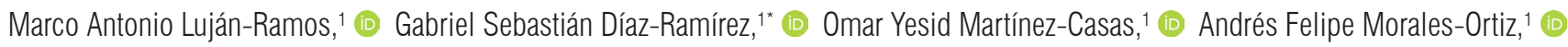
Jorge Hernando Donado-Gómez, ${ }^{2}$ (D) Juan Carlos Restrepo-Gutiérrez, ${ }^{3}$ (ib) Juan Ignacio Marín-Zuluaga. ${ }^{4}$ (i)

\author{
Gacceso abierto \\ Citación: \\ Luján-Ramos MA, Díaz-Ramírez GS, Martínez- \\ Casas OY, Morales-Ortiz AF, Donado-Gómez \\ $\mathrm{JH}$, Restrepo-Gutiérrez JC, Marin-Zuluaga \\ Jl. Caracterización de pacientes con cirrosis \\ hepática y bacteriemia de un hospital \\ universitario en Medellín, Colombia. Rev Colomb \\ Gastroenterol. 2020;35(4):455-464. https://doi. \\ org/10.22516/25007440.557
}

Hepatología clínica, Universidad de Antioquia, Medellín, Colombia.

Unidad de epidemiología, Hospital Pablo Tobón Uribe. Profesor de la Universidad de Antioquia. Medellín, Colombia.

Hepatología clínica, Universidad de Antioquia. Unidad de hepatología y trasplante hepático, Hospital Pablo Tobón Uribe. Medellín, Colombia.

Unidad de hepatología y trasplante hepático,

Hospital Pablo Tobón Uribe. Medellín, Colombia.

*Correspondencia: Gabriel Sebastián Díaz Ramírez. sebastiandiazr@gmail.com

Fecha recibido: $\quad 03 / 05 / 20$ Fecha aceptado: 08/07/20

\section{Resumen}

Introducción: la bacteriemia en pacientes cirróticos es frecuente y se asocia con una alta mortalidad y hospitalización prolongada. Este estudio describe las características demográficas, clínicas y de laboratorio en pacientes con cirrosis hepática y bacteriemia en un hospital de cuarto nivel. Métodos: estudio observacional de cohorte retrospectiva. Incluyó pacientes con cirrosis hepática y bacteriemia entre el 1 de enero de 2010 y el 31 de diciembre de 2017 en el Hospital Pablo Tobón Uribe de Medellín, Colombia. Se recogieron variables demográficas, clínicas y de laboratorio. Se estimó la supervivencia durante el tiempo de hospitalización y hasta 30 días desde el diagnóstico de bacteriemia. Resultados: se hallaron 78 pacientes con cirrosis y bacteriemia. La media de edad fue de 65 años, 66,7 \% fueron mujeres. Las principales etiologías de la cirrosis fueron: criptogénica $(30,8 \%$ ) y esteatohepatitis no alcohólica (EHNA; 19,3\%). La principal fuente de infección fue la vía urinaria (24\%), seguida de colangitis $(23 \%)$ y la bacteriemia espontánea (19\%). Los bacilos gramnegativos (BGN) representaron la mayoría de los aislamientos $(67,9 \%)$. La prevalencia de multidrogorresistentes (MDR) fue de 25,6 \% y el uso adecuado de antibiótico empírico fue de 80,8 \%. La mortalidad a 30 días fue de 11,5\%. Como mejores predictores de mortalidad se encontraron la puntuación Child-Pugh y Model for End-stage Liver Disease (MELD) al ingreso con área bajo la curva ROC (AUROC) de $0,79(p=0,008)$ y $0.72(p=0,042)$, respectivamente. Conclusiones: los hallazgos permiten conocer las principales características de los pacientes con cirrosis que desarrollan bacteriemia en nuestro medio. Se encontró un número considerable de infecciones MDR. Los pacientes con un grado avanzado de la cirrosis son los que presentan un mayor riesgo de mortalidad.

\section{Palabras clave}

Cirrosis hepática, bacteriemia, Colombia.

\begin{abstract}
Introduction: Bacteremia in cirrhotic patients is frequent and associated with high mortality and prolonged hospital stays. This study describes the demographic, clinical, and laboratory characteristics of patients with liver cirrhosis and bacteremia treated at a quaternary care hospital. Methodology: Observational, retrospective cohort study. The sample consisted of patients with liver cirrhosis and bacteremia treated between January 1 , 2010, and December 31, 2017, at the Hospital Pablo Tobon Uribe of Medellín, Colombia. Demographic, clinical, and laboratory variables were collected. Survival was estimated during the time of hospitalization and up to 30 days following the diagnosis of bacteremia. Results: 78 patients had cirrhosis and bacteremia. The average age was 65 years; $66.7 \%$ were women. Cirrhosis was labeled cryptogenic in $30.8 \%$ of the cases and NASH in $19.3 \%$. The main source of infection was the urinary tract $(24 \%)$, followed by cholangitis $(23 \%)$ and spontaneous bacteremia $(19 \%)$. Gram-negative bacteria were observed in most of the isolates $(67.9 \%)$. The prevalence of MDR was $25.6 \%$, and the adequate use of empirical antibiotics was $80.8 \%$. The 30 -day mortality rate was $11.5 \%$. The best mortality predictors were the Child-Pugh and MELD scores on admission with AUROC of $0.79(P=0.008)$ and $0.72(P=0.042)$, respectively. Conclusions: The findings allow describing the main characteristics of patients with cirrhosis who develop bacteremia in our environment. A considerable number of MDR infections were found. Patients with an advanced degree of cirrhosis are at the highest risk of mortality.
\end{abstract}

Keywords

Liver Cirrhosis; Bacteremia; Colombia. 


\section{INTRODUCCIÓN}

La cirrosis hepática es la décima causa de muerte en el mundo occidental (1). Las infecciones son complicaciones importantes en cirróticos en términos de incidencia, gravedad y desenlaces globales (2), representan la causa principal de admisión a urgencias de estos pacientes. Cerca del $20 \%$ $30 \%$ de las admisiones hospitalarias por descompensación de la cirrosis están relacionadas con una infección, o se complican con una infección durante la hospitalización (2-4). Adicionalmente, la necesidad de exposición frecuente al ambiente hospitalario, por las complicaciones de la cirrosis, hacen que esta población sea susceptible de infecciones asociadas con el cuidado de la salud, caracterizadas por patógenos multidrogorresistentes (MDR) $(3,5,6)$.

La mortalidad relacionada con infecciones cirróticas se acerca con el $30 \%$ en los primeros 30 días y $66 \%$ a 1 año desde el ingreso al hospital, por lo que son consideradas un factor pronóstico importante en pacientes con enfermedad hepática terminal (7).

La bacteriemia es frecuente en los pacientes con cirrosis, afecta a un $4 \%-21 \%$ de estos y es hasta 10 veces más frecuente en pacientes cirróticos que en no cirróticos $(8$, 9). La bacteriemia en cirróticos se asocia con una alta mortalidad, hospitalización prolongada y deterioro de la enfermedad hepática $(10,11)$. En diferentes estudios de pacientes con bacteriemia o candidemia se ha encontrado que la cirrosis hepática es un predictor independiente de mortalidad (12-14).

Pocos estudios han investigado las características y los factores de riesgo específicos de mortalidad en pacientes con bacteriemia y cirrosis. La mayoría de la evidencia disponible proviene de estudios unicéntricos, especialmente en Europa y Asia.

En Argentina, un estudio de Marciano y colaboradores encontró que las tasas de insuficiencia renal aguda y mortalidad de los pacientes con bacteriemia espontánea eran similares a las de los pacientes con peritonitis espontánea (15). Se desconoce el comportamiento de esta entidad, especialmente en Colombia. El objetivo del presente estudio es describir las características demográficas, clínicas y de laboratorio de una cohorte de pacientes con cirrosis hepática y bacteriemia en un Hospital Universitario de la ciudad de Medellín, Colombia; asimismo, describir la supervivencia a 30 días, los factores relacionados con la misma y los agentes patógenos aislados, su perfil de resistencia y la susceptibilidad al tratamiento antibiótico empírico utilizado.

\section{MÉTODOS}

Estudio observacional de cohorte retrospectiva. Incluyó a pacientes atendidos entre el 1 de enero de 2010 al 31 de diciem- bre de 2017 en el servicio de hospitalización del Hospital Pablo Tobón Uribe (HPTU) en Medellín, Colombia.

Se realizó una búsqueda en el sistema de historia clínica electrónica de pacientes con diagnóstico de cirrosis hepática (códigos K703, K743, K744, K745, K 746, K717) y bacteriemia (código A49.9) según la Clasificación Internacional de Enfermedades (CIE-10). Los criterios de inclusión fueron: pacientes mayores de 18 años con diagnóstico de cirrosis hepática y pacientes con bacteriemia diagnosticados durante el período de hospitalización. Se excluyeron pacientes sin un diagnóstico claro de cirrosis y aquellos sin reporte de hemocultivos en la historia clínica.

Se midieron variables demográficas, clínicas y de laboratorio. El diagnóstico de cirrosis hepática fue establecido por histología o por hallazgos clínicos, bioquímicos o radiológicos. Las complicaciones no infecciosas de la cirrosis (ascitis, várices esofágicas, síndrome hepatorrenal, encefalopatía hepática, hepatocarcinoma) se definieron usando los criterios de la Asociación Europea para el Estudio del Hígado (EASL) y el Club Internacional de la Ascitis (16).

La presencia de bacteriemia se definió por el aislamiento de un microorganismo no contaminante de la piel en 1 o más hemocultivos, o el aislamiento de un microorganismo contaminante común de la piel (difteroides, Bacillus spp, Propionibacterium spp, estafilococos coagulasa negativo o micrococo) en 2 o más hemocultivos. Se clasificó como adquirida en la comunidad, asociada con los cuidados de la salud, o nosocomial, de acuerdo con los criterios de Friedman (17). Se consideró bacteriemia espontánea ante la presencia de hemocultivos positivos (descartando contaminación) en ausencia de una fuente de infección.

La fuente de la infección se determinó según lo establecido en la historia clínica. El diagnóstico de peritonitis bacteriana espontánea (PBE) se definió como la presencia de 250 o más polimorfonucleares en líquido ascítico, con o sin aislamiento microbiológico. Si el paciente presentaba este diagnóstico, se consideraba como el origen de la bacteriemia cuando no había identificación de otro foco infeccioso.

Los microorganismos aislados se clasificaron de acuerdo con su susceptibilidad en multidrogorresistentes (MDR) o no MDR, según las determinaciones de la European Society of Clinical Microbiology and Infectious Diseases (ESCMID), en las que define MDR como la ausencia de susceptibilidad de al menos un agente en 3 o más categorías antibióticas (18).

Los datos se recolectaron mediante la revisión de historias clínicas con un instrumento de recolección previamente diseñado. Para asegurar la calidad de los datos y disminuir el riesgo de sesgo de información, se revisó el $10 \%$ de las historias clínicas en duplicado entre los investigadores. Se realizó el seguimiento de los pacientes hasta 30 días luego del diagnóstico de bacteriemia, o hasta la última fecha documentada en la historia clínica. 
Las variables categóricas se presentan como frecuencias absolutas y relativas. Las variables continuas como media y desviación estándar ( $\mathrm{DE}$ ) según tengan una distribución normal o mediana, y rango intercuartílico (RIC) en aquellas sin distribución normal según la prueba de Shapiro-Wilk. Se calculó la densidad de la incidencia de mortalidad en los pacientes con cirrosis hepática y bacteriemia del siguiente modo: número de pacientes con mortalidad dividido por la sumatoria del tiempo de seguimiento de todos los pacientes.

Se estimó la supervivencia por el método de KaplanMeier durante el tiempo de hospitalización, según las variables que por plausibilidad biológica (como son el puntaje Child-Pugh, Model for End-stage Liver Disease [MELD], grado de insuficiencia hepática aguda sobre crónica [ACLF], entre otras) se han asociado con mortalidad, según los diferentes estudios. Como análisis exploratorio, se determinó el rendimiento de diferentes variables en la predicción de mortalidad durante la hospitalización al ingreso, al momento de la bacteriemia y 24 y 48 horas después. Se empleó el paquete estadístico SPSS versión registrada 20.0 de la Universidad de Antioquia.

No se estimó un tamaño de muestra a priori, ya que se incluyeron todos los pacientes con cirrosis hepática y diagnóstico de bacteriemia atendidos durante el período de estudio.

Este estudio contó con la aprobación del comité de ética del HPTU, se siguieron las normas sobre aspectos éticos de la investigación clínica contenidas en la Resolución 008430 de 1993 del Ministerio de Protección Social de la República de Colombia y se adhirió a los lineamientos de la declaración de Helsinki, versión 2013, para la investigación en seres humanos. El manuscrito final se adhirió a las recomendaciones de Strengthening the Reporting of Observational studies in Epidemiology (STROBE) para el reporte de estudios observacionales (19).

\section{RESULTADOS}

Se hallaron 78 pacientes con cirrosis hepática y bacteriemia (Tabla 1). La media de edad fue de 65 años. El 66,7 \% de la población fue de sexo femenino. Las principales etiologías de cirrosis fueron criptogénica $(30,8 \%)$, esteatohepatitis no alcohólica (EHNA; 19,2 \%) y alcohol (19,2\%). 8 pacientes $(10,3 \%)$ no presentaban otros antecedentes o comorbilidades aparte de la cirrosis, $19 \%$ presentaba al menos una comorbilidad y $71 \%$ presentaba dos o más; la hipertensión arterial (HTA) en el 44,9\% (35 casos), la diabetes en el $37,2 \%$ (29 casos) y la obesidad o sobrepeso en el $14,1 \%$ (11 casos) fueron las más comunes. En relación con la gravedad de la cirrosis, $17,9 \%$ (14 pacientes), $53,3 \%$ (42 pacientes) y $28,2 \%$ (22 pacientes) tuvieron clase A, B y C, respectivamente, según la clasificación Child-Pugh, con un puntaje MELD promedio al ingreso de 15,1.
Tabla 1. Características de los 78 pacientes con cirrosis y bacteriemia confirmada

\begin{tabular}{|c|c|}
\hline & $n(\%)$ \\
\hline Edad (años) & $65,18( \pm 13,13)$ \\
\hline Sexo femenino $(\%)$ & $52(66,7)$ \\
\hline $\begin{array}{l}\text { Raza (mestiza/blanca/negra) } \\
\text { - } \quad \text { Mestiza } \\
\text { - } \text { Blanca } \\
\text { - } \quad \text { Negra }\end{array}$ & $\begin{array}{l}71(91) \\
4(5,1) \\
3(3,8)\end{array}$ \\
\hline 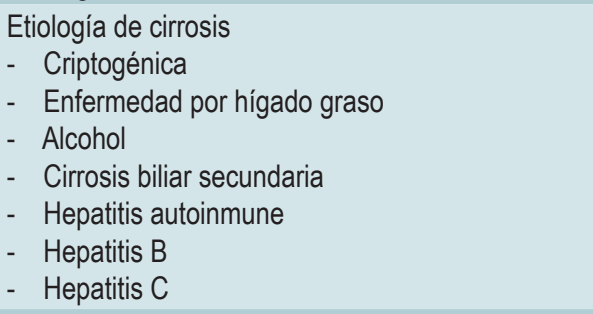 & $\begin{array}{l}24(30,8)^{*} \\
15(19,2) \\
15(19,2) \\
13(16,7) \\
5(6,4) \\
2(2,6) \\
4(5,1)\end{array}$ \\
\hline $\begin{array}{l}\text { Estadio Child-Pugh } \\
-\quad \text { A } \\
-\quad \text { B } \\
-\quad \text { C }\end{array}$ & $\begin{array}{l}14(17,9) \\
42(53,3) \\
22(28,2)\end{array}$ \\
\hline Puntaje MELD, mediana (RIC) & $14(9,1-20,3)$ \\
\hline $\begin{array}{l}\text { Complicaciones cirróticas previas a hospitalización } \\
\text { - Várices esofágicas } \\
\text { - Sangrado varicoso } \\
\text { - Encefalopatía hepática } \\
\text { - Ascitis } \\
\text { - Hepatocarcinoma } \\
\text { - Peritonitis bacteriana espontánea } \\
\text { - Trombosis portal } \\
\text { - Síndrome hepatorrenal }\end{array}$ & $\begin{array}{l}34(43,6) \\
19(24,4) \\
31(39,7) \\
22(28,2) \\
14(17,6) \\
12(15,4) \\
10(12,8) \\
5(6,4)\end{array}$ \\
\hline 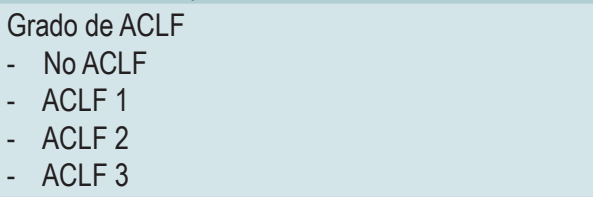 & $\begin{array}{l}57(73,1) \\
10(12,8) \\
6(7,7) \\
5(6,4)\end{array}$ \\
\hline Días de hospitalización, mediana (RIC) & $11(5-15)$ \\
\hline $\begin{array}{l}\text { Admisión a una unidad de cuidado crítico } \\
\text { - UCI } \\
\text { - UCE }\end{array}$ & $\begin{array}{l}4(5,1) \\
18(23,1)\end{array}$ \\
\hline $\begin{array}{l}\text { Fuente de la infección } \\
\text { - } \text { Comunidad } \\
\text { - IAAS } \\
\text { - } \quad \text { Nosocomial }\end{array}$ & $\begin{array}{l}34(43,6) \\
29(37,2) \\
15(19,2)\end{array}$ \\
\hline 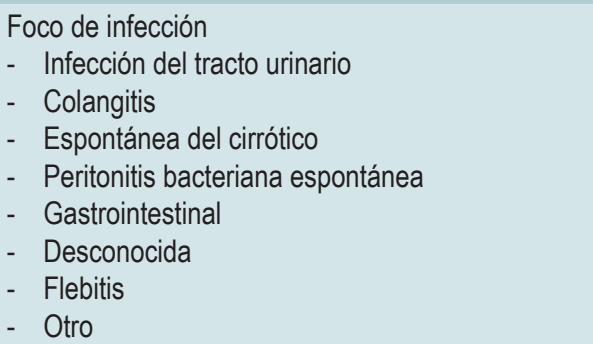 & $\begin{array}{l}19(24,4) \\
18(23,1) \\
15(19,2) \\
12(15,5) \\
4(5,1) \\
3(3,8) \\
2(2,6) \\
5(6,4)\end{array}$ \\
\hline
\end{tabular}

*La suma no alcanza el $100 \%$ dado que en los otros casos la etiología no es clara. ACLF: insuficiencia hepática aguda sobre crónica; IAAS: infección asociada con el cuidado de la salud; UCE: unidad de cuidados especiales; UCI: unidad de cuidados intensivos. 
Los principales focos de bacteriemia fueron infecciones del tracto urinario en el $24,4 \%$ (19 casos), colangitis en el 23,1 \% (18 casos), bacteriemia espontánea en el 19,2\% (15 casos) y PBE en el 15,4 \% (12 casos). Las infecciones adquiridas en comunidad fueron la principal fuente de infección (43,6 \%; 34 casos), seguidas de las infecciones asociadas con el cuidado de la salud $(37,2 \% ; 29$ casos) y las nosocomiales (19,2\%; 15 casos). La media de tiempo para el reporte de cultivo positivo desde el ingreso hospitalario fue de 1,8 días (rango: 0-34).

Los bacilos gramnegativos (BGN) representaron la mayor parte (53 casos; 67,9 \%); de estos, la Escherichia coli fue la más frecuente, con 36 casos (46,2\%); seguida por Klebsiella pneumoniae, con 8 casos (10,3\%). Los cocos grampositivos (CGP) constituyeron el 30,8 \% (24 casos), y el género Streptococcus fue el más frecuente (24,4\%; 19 casos). En dos casos (2,6\%) se hallaron 2 o más bacterias, ambos en sobrevivientes; y en un paciente que falleció, además de BGN, se halló Candida tropicalis. La prevalencia de MDR fue 25,6\% (20 casos), a destacar 5 casos de $E$. coli productoras de betalactamasas de espectro extendido (BLEE), dos E. coli productoras de betalactamasa AmpC, una K. pneumoniae BLEE y tres Staphylococcus aureus resistentes a meticilina (Tabla 2).

\section{Análisis exploratorio de variables relacionadas con mortalidad}

La mortalidad fue del $11,5 \%$. La mediana del puntaje MELD fue 13 (RIC: 8,9-19,9) y 14,57 (RIC: 9,1-19,1) en sobrevivientes frente a 21,06 (RIC: $12,5-27,2$ ) y 20,26 (RIC: 11-23) en quienes murieron al ingreso y al diagnóstico de bacteriemia, respectivamente; con diferencias estadísticamente significativas al ingreso $(p=0,041)$ (Tabla 3, Figura 1). La media de tiempo de hospitalización fue de 11 días (RIC: 1-21) y 13 días (RIC: 1-24) en sobrevivientes y quienes fallecieron, respectivamente, sin diferencias significativas $(p=0,573)$.

Las infecciones del tracto urinario $(26,5 \%)$, bacteriemia espontánea $(20,3 \%)$ y colangitis $(23,5 \%)$ fueronlas más frecuentes en sobrevivientes; a diferencia de aquellos que perecieron, en quienes la PBE $(33,3 \%)$ y la colangitis $(22,2 \%)$ fueron las más reportadas, aunque sin diferencias estadísticamente significativas (Figura 2). La mortalidad en bacteriemia por gramnegativos fue del $13,5 \%$ y del $7,7 \%$ en grampositivos, diferencia estadísticamente no significativa $(p=0,453)$. El uso adecuado de antibiótico empírico fue del $80,8 \%$, sin diferencia entre los pacientes sobrevivientes frente a los que fallecieron $(79,4 \%$ y $88,9 \%$, respectivamente). No hubo diferencias significativas en cuanto al tipo de aislamiento bacteriano ni el perfil MDR según vivos o muertos; sin embargo, en quienes murieron, la fuente de
Tabla 2. Microorganismos aislados en hemocultivos en pacientes con cirrosis hepática y bacteriemia

\begin{tabular}{|c|c|c|c|}
\hline Tipo de germen & $n$ & $\begin{array}{l}\text { Porcentaje } \\
\text { total }\end{array}$ & $\begin{array}{c}\text { MDR } \\
(\%)\end{array}$ \\
\hline $\begin{array}{l}\text { Gramnegativos } \\
\text { - E. coli } \\
\text { - K. pneumoniae } \\
\text { - Klebsiella oxytoca } \\
\text { - Aeromonas spp. } \\
\text { - Burkholderia cepacia } \\
\text { - Proteus mirabilis } \\
\text { - Salmonella enterica } \\
\text { - Citrobacter freundii } \\
\text { - Pseudomonas oleovorans } \\
\text { - Pantoea spp }\end{array}$ & $\begin{array}{c}36 \\
8 \\
2 \\
2 \\
1 \\
1 \\
1 \\
1 \\
1 \\
1\end{array}$ & $\begin{array}{c}46,2 \\
10,2 \\
2,5 \\
2,5 \\
1,3 \\
1,3 \\
1,3 \\
1,3 \\
1,3 \\
1,3\end{array}$ & $\begin{array}{c}35 \\
12,5 \\
0 \\
0\end{array}$ \\
\hline $\begin{array}{l}\text { CGP } \\
\text { - S. aureus } \\
\text { - Staphylococcus coagulasa negativo } \\
\text { - Streptococcus salivarius } \\
\text { - Streptococcus agalactiae } \\
\text { - Streptococcus mitis } \\
\text { - Streptococcus pneumoniae } \\
\text { - Streptococcus sanguis } \\
\text { - Streptococcus gordonii } \\
\text { - Streptococcus infantarius }\end{array}$ & $\begin{array}{l}4 \\
2 \\
4 \\
4 \\
3 \\
3 \\
2 \\
1 \\
1\end{array}$ & $\begin{array}{l}5,1 \\
2,5 \\
5,1 \\
5,1 \\
3,8 \\
3,8 \\
2,5 \\
1,3 \\
1,3\end{array}$ & $\begin{array}{c}75 \\
100\end{array}$ \\
\hline $\begin{array}{l}\text { BGP } \\
-\quad \text { Listeria monocytogenes }\end{array}$ & 1 & 1,3 & 0 \\
\hline $\begin{array}{l}\text { Hongos } \\
\text { - Candida albicans }\end{array}$ & 1 & 1,3 & 0 \\
\hline $\begin{array}{l}\text { Resistencia } \\
\text { - No MDR } \\
\text { - MDR }\end{array}$ & $\begin{array}{l}58 \\
20\end{array}$ & $\begin{array}{l}74,4 \\
25,6\end{array}$ & \\
\hline
\end{tabular}

infección asociada con el cuidado de la salud fue mayor con diferencia significativa $(31,1 \%$ frente a $77,8 \% ; p=0,007)$.

En cuanto al grado de insuficiencia hepática aguda sobre crónica (ACLF), 10 (12,8\%), 6 (7,7\%) y 5 pacientes $(6,4 \%)$ presentaron ACLF grados 1,2 y 3 , respectivamente, al ingreso, con tasas de mortalidad de $40 \%$ y $50 \%$ para los dos últimos grados, $7 \%$ en no ACLF y ningún caso de mortalidad en ACLF 1; con diferencias y asociación estadísticamente significativas en la mortalidad entre no ACLF y ACLF 1 frente a ACLF 2 y 3 (4/66 frente a $5 / 11 ; p=$ $0,0001)$. Al considerar la gravedad de la cirrosis, se halló una mortalidad significativamente más alta a mayor clase Child-Pugh $(p=0,037)$ y, aunque también hubo tendencia según el grado ACLF, esta diferencia no fue significativa $(p$ $=0,09$ ) (Figuras 3 y 4 ).

Como mejores predictores de mortalidad se encontraron la puntuación Child-Pugh y MELD al ingreso con áreas 
Tabla 3. Comparación de las condiciones basales al ingreso: sobrevivientes frente a no sobrevivientes

Sobrevivientes, $\mathrm{n}=69$

No sobrevivientes, $\mathbf{n}=\mathbf{9}$

Valor $p$

Datos demográficos

- Edad, media (DE)

- Sexo femenino

- Raza mestiza

$66( \pm 11,45)$

$45(65,2 \%)$

$63(91,3)$

Etiología

- Criptogénica

- Enfermedad por hígado graso

- Relacionada con alcohol

- Hepatitis autoinmune

- Biliar secundaria

- Hepatitis B

- Hepatitis C

- MELD, mediana, RIC

Complicaciones previas a la hospitalización, $n$ (\%)

- Trombosis portal

- Hepatocarcinoma

- Ascitis

- PBE

- SHR

- Encefalopatía

- Sangrado varicoso

Datos de bacteriemia

- Días hasta cultivo positivo

Fuente de infección

- Comunidad

- IACS

- Nosocomial

Foco infeccioso

- PBE

- Colangitis

- Espontanea

- Desconocida

- Gastrointestinal

- ITU

- Flebitis

- Hospitalización en días, mediana (RIC)
$19(27,5 \%)$

$15(21,7 \%)$

$15(21,7 \%)$

$4(5,8 \%)$

$11(15,9 \%)$

$1(1,4 \%)$

$4(5,8 \%)$

$13(8,9-19,9)$

$7(10,3 \%)$

$14(20,5 \%)$

$16(23,5 \%)$

$10(14,7 \%)$

$4(5,9 \%)$

$26(38,2 \%)$

$16(32,5 \%)$

$1,78( \pm 5)$

$33(47,8 \%)$

$22(31,9 \%)$

$14(20,3 \%)$

$9(13 \%)$

$16(23,2 \%)$

$14(20,3 \%)$

$3(4,3)$

$4(5,8 \%)$

$18(26,1 \%)$

$2(2,9 \%)$

$11(6,2-16,5)$

\section{$58,6( \pm 22,3)$ \\ $7(77,8 \%)$ \\ $8(88,9 \%)$}

0,114

0,453

0,810

$5(55,6 \%)$

0,087

0

0

$1(11,1 \%)$

$2(22,2 \%)$

$1(11,1)$

0

$21(12,5-21,7)$

0,118

0,118

0,541

0,541

0,085

0,541

$0,041^{*}$

$3(33,3)$

0

$5(55,6 \%)$

$2(22,2 \%)$

$1(11,1 \%)$

$4(44 \%)$

$3(33,3 \%)$

0,053

0,133

0,043

0,559

0,550

0,720

$0,52,1$

$1,89( \pm 3,8)$

0,950

*Diferencia de medianas. IACS: infección asociada con el cuidado de la salud; ITU: infección del tracto urinario; SHR: síndrome hepatorrenal.

bajo la curva ROC de 0,79 ( $p=0,008$; intervalo de confianza [IC] $95 \%$ : 0,63-0,95) y $0,72(p=0,042$; IC $95 \%$ : $0,45-0,89)$, respectivamente (Figura 5).

\section{DISCUSIÓN}

A nuestro conocimiento, este estudio es el más grande publicado en América Latina en su tipo. La mayoría de los estudios sobre infección en pacientes cirróticos ha analizado a pacientes con $\mathrm{PBE}$, solo algunos pocos han descrito las características epidemiológicas de bacteriemia en esta población. Dos de los estudios más grandes sobre incidencia, distribución etiológica y desenlaces de bacteriemia en pacientes cirróticos se publicaron hace más de una década $(9,20)$. En América Latina se destaca un estudio realizado en Chile en el que reportaron las características de 59 pacientes con bacteriemia asociado con daño hepático crónico, con predominio de infecciones por CGP y con una mortalidad del $37 \%$ (21); 


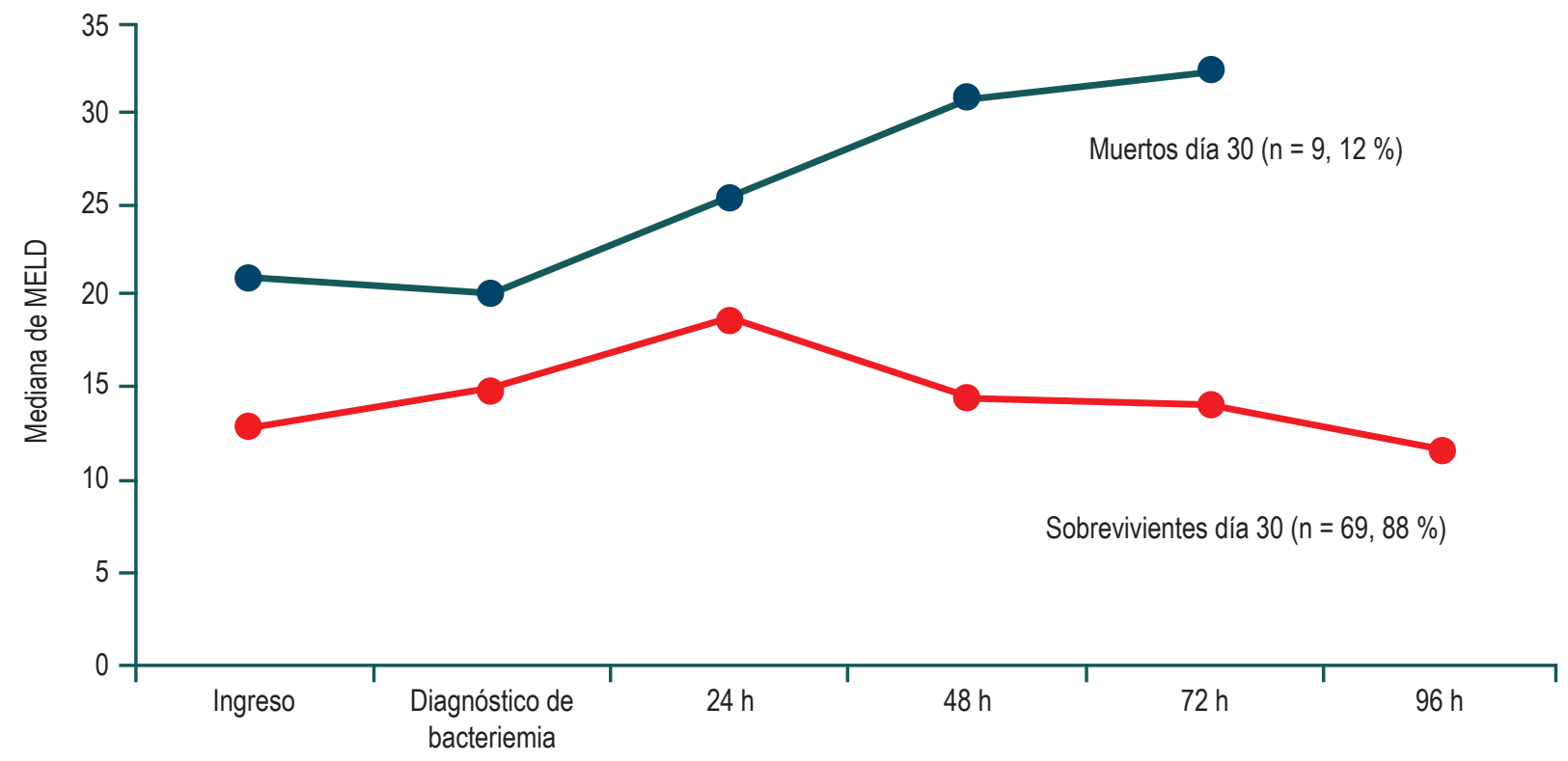

Tiempo de valoración

Figura 1. Patrón de la mediana en la puntuación MELD de pacientes cirróticos con bacteriemia desde el ingreso hospitalario en sobrevivientes (línea verde) y muertos (línea azul) a 30 días de haber ingresado.

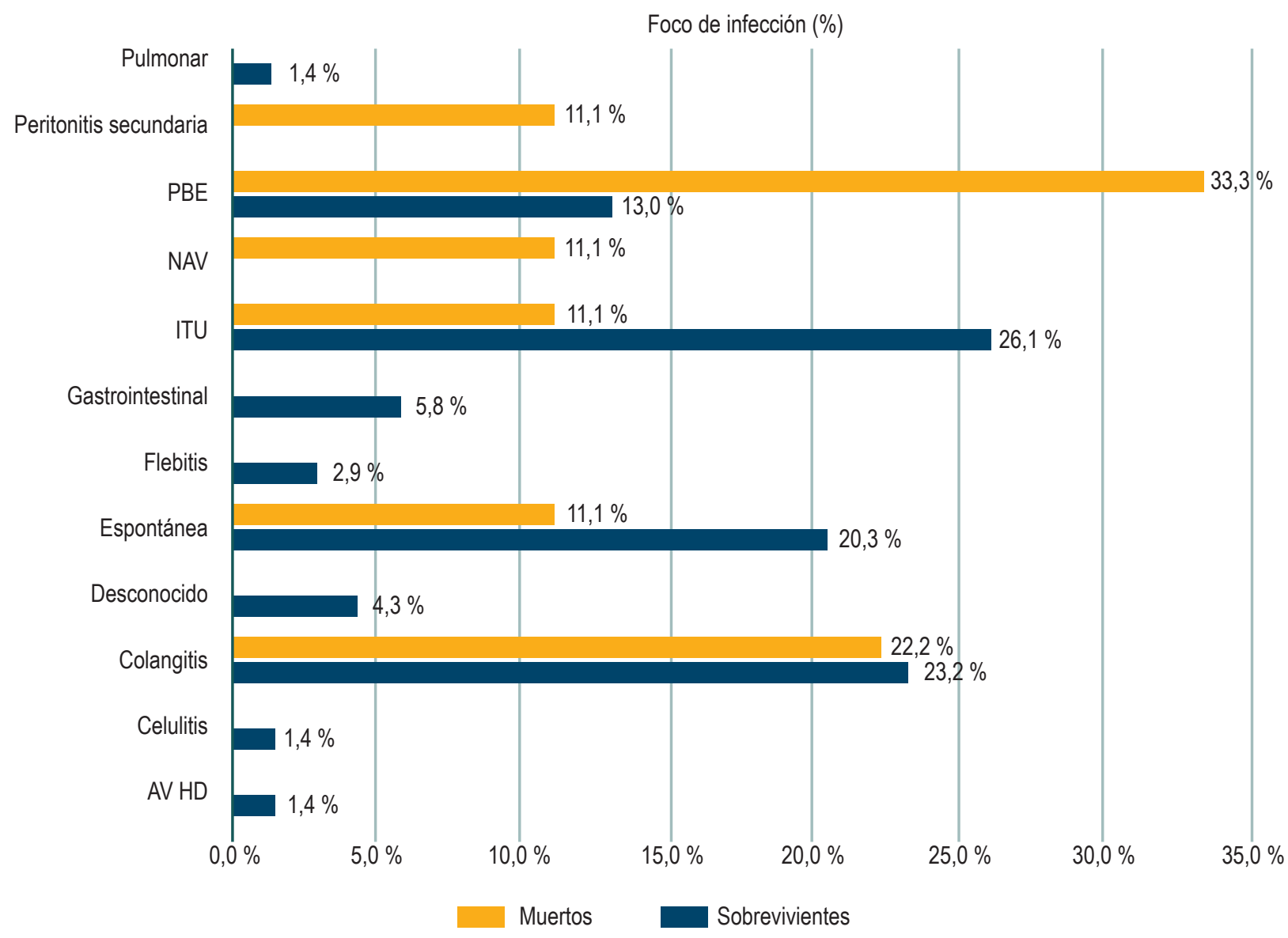

Figura 2. Frecuencia de focos clínicos de bacteriemia en pacientes cirróticos sobrevivientes y muertos a 30 días de la infección. AV HD: acceso vascular de hemodiálisis; NAV: neumonía asociada con el ventilador. 


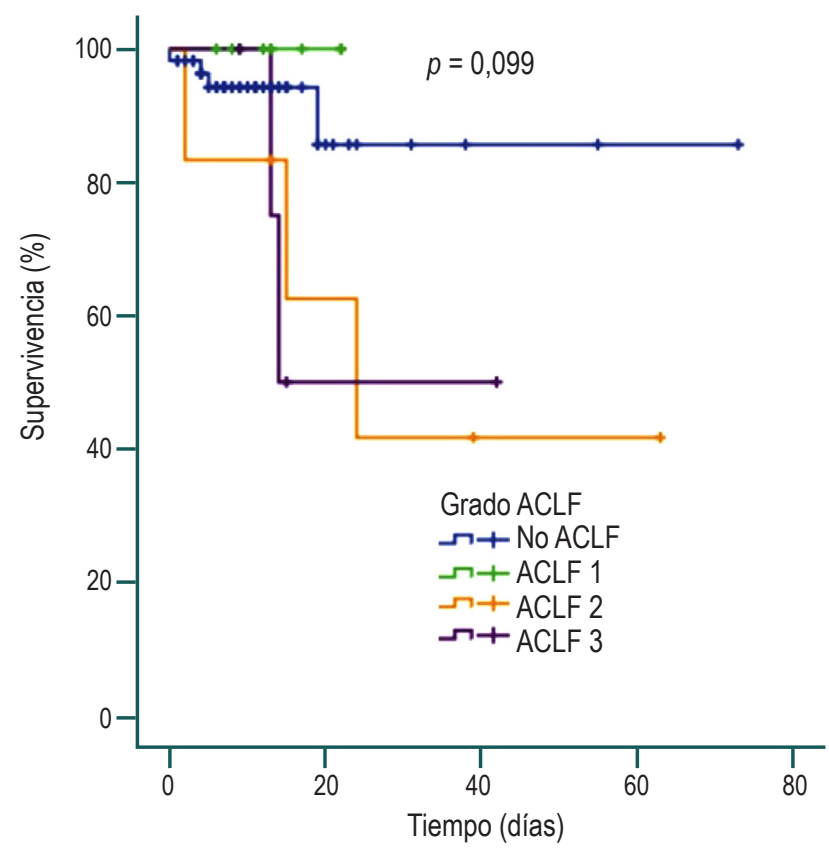

Figura 3. Curva de supervivencia en pacientes con cirrosis y bacteriemia según el grado ACLF.

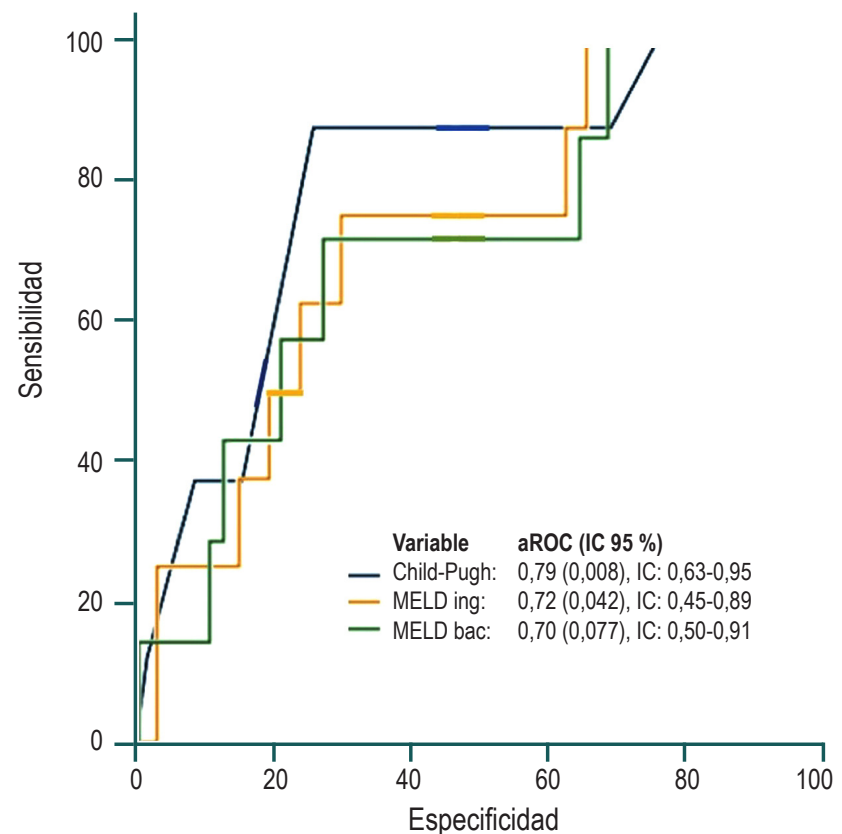

Figura 5. Comparación en el área bajo la curva ROC entre variables como predictores de supervivencia al egreso.

y un estudio en Argentina en el que la bacteriemia espontánea tuvo similares tasas de mortalidad e insuficiencia renal que la peritonitis espontánea (15).

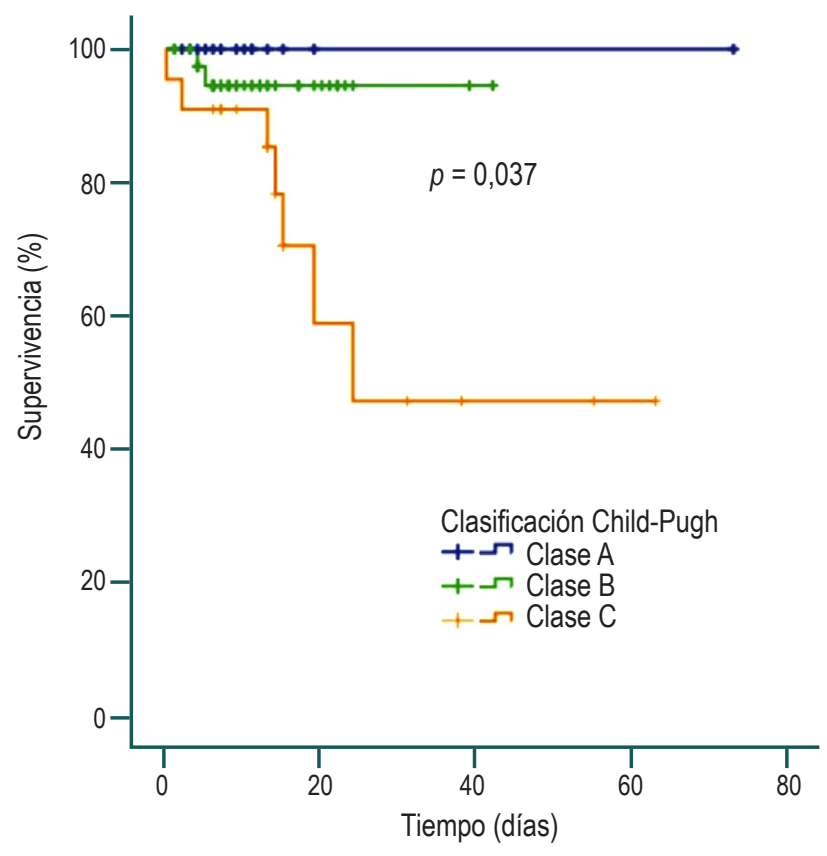

Figura 4. Curva de supervivencia en pacientes con cirrosis y bacteriemia según la clasificación Child-Pugh.

En el presente estudio se encontró un alto porcentaje de cirrosis criptogénica y por EHNA, asociado con una alta tasa de comorbilidades relacionadas con el síndrome metabólico, lo que sugiere que algunos de los pacientes catalogados como cirrosis criptogénica fueran realmente EHNA; sin embargo, la realización de biopsia hepática no estaría indicada ya que no cambiaría el abordaje terapéutico ni el pronóstico hepático. La mayoría de los eventos se registraron en pacientes cirrosis Child-Pugh clase B y hasta en un $20 \%$ de los casos fue necesario el ingreso a la unidad de cuidados intensivos (UCI) para el manejo y monitorización de su enfermedad. La principal fuente de infección fue la vía urinaria, vía biliar, PBE y bacteriemia espontánea del cirrótico, lo cual es acorde con las principales complicaciones infecciosas descritas en pacientes con enfermedad hepática terminal, favorecida por la inmunosupresión determinada por la insuficiencia hepática, entre otros factores (22).

La mortalidad a 30 días fue de un 11,5\%, que es mucho menor en comparación con lo reportado en otros estudios $(15,22-26)$, probablemente porque los pacientes de este estudio tenían menos disfunción hepática ( $28 \%$ en Child-Pugh clase C) y el $73 \%$ no desarrollaron ACLF. La mediana de MELD al momento del ingreso y del diagnóstico de bacteriemia fue mucho mayor en los pacientes que fallecieron frente a los sobrevivientes, con diferencia estadísticamente significativa. En la población con desenlace fatal se encontró una mayor disfunción hepática, según la 
clasificación de Child-Pugh, y un mayor grado de ACLF con diferencia estadísticamente significativa cuando se compararon los grados 2 y 3 frente al grado 1 y el grupo no ACLF, y la puntuación de Child-Pugh y MELD fueron los mejores predictores de mortalidad. Otros autores han reportado que variables como el delta MELD (24, 48 y 72 horas), PBE como fuente de la infección, antibioticoterapia empírica inapropiada y presencia de sepsis grave o choque séptico se asociaron con una mayor mortalidad a 30 días (23). Consideramos que, debido a la baja cantidad de pacientes fallecidos a 30 días y a la ausencia de datos para el cálculo del delta MELD, no fue posible establecer dicha relación en el presente estudio.

Las infecciones por BGN representaron más del $66 \%$ de los casos, y la E. coli es el principal patógeno con un $46 \%$ del total y una tasa de MDR de $35 \%$; llamativamente, el $75 \%$ y $100 \%$ de las infecciones por S. aureus y S. coagulasa negativo, respectivamente, fueron por un germen MDR (con resistencia a meticilina). En nuestra institución, el cubrimiento antibiótico empírico de los pacientes cirróticos con infecciones adquiridas en la comunidad se realiza con piperacilina/tazobactam y con meropenem ante infecciones asociadas con los cuidados de la salud o nosocomiales (+/- vancomicina de acuerdo con la sospecha de CGP resistente), lo cual se traduce en el amplio porcentaje reportado de cubrimiento empírico apropiado (más del $80 \%$ ).

El presente estudio presenta algunas limitaciones. Es un estudio unicéntrico; por tanto, los datos presentados pueden no ser representativos de otras instituciones. Sin embargo, nuestro hospital es un centro de referencia para pacientes con enfermedades hepáticas, con muchos pacientes provenientes de diferentes regiones del país. No se evaluó el desarrollo de insuficiencia renal aguda, la cual ha demostrado tener impacto a corto plazo en este grupo de pacientes (15). Adicionalmente, la cantidad de eventos es limitada, principalmente los casos con mortalidad a 30 días, lo cual dificulta establecer mayores predictores de desenlace fatal en esta población.

\section{CONCLUSIÓN}

El presente estudio no permite conocer las principales características de los pacientes con cirrosis hepática que desarrollan bacteriemia en nuestro medio. El estudio etiológico revela cuáles son los principales gérmenes que afectan esta población con una cantidad considerable de infecciones MDR que viene en aumento no solo en nuestro medio, sino también a nivel mundial y que limita cada vez más la forma en que tratamos a nuestros pacientes. Los pacientes con un grado avanzado de su enfermedad hepática crónica al momento de la hospitalización y del diagnóstico de bacteriemia, al igual que un grado alto de ACLF (2 o 3), son los pacientes que presentan un mayor riesgo de desenlace fatal y que, por tanto, requieren un mayor cuidado en su atención y manejo.

\section{Fuente de financiación}

Propia.

\section{Conflictos de interés}

Los autores manifiestan no tener ningún conflicto de interés.

\section{REFERENCIAS}

1. Leon DA, McCambridge J. Liver cirrhosis mortality rates in Britain from 1950 to 2002: an analysis of routine data. Lancet. 2006;367(9504):52-6. http://dx.doi. org/10.1016/S0140-6736(06)67924-5

2. Fernandez J, Gustot T. Management of bacterial infections in cirrhosis. J Hepatol. 2012;56 Suppl 1:S1-12. http://dx.doi.org/10.1016/S0168-8278 (12)60002-6

3. Fernandez J, Acevedo J, Castro M, Garcia O, de Lope CR, Roca D, Pavesi M, Sola E, Moreira L, Silva A, Seva-Pereira T, Corradi F, Mensa J, Ginès P, Arroyo V. Prevalence and risk factors of infections by multiresistant bacteria in cirrhosis: a prospective study. Hepatology. 2012;55(5):1551-61. http://dx.doi.org/10.1002/hep.25532

4. Pant C, Olyaee M, Gilroy R, Pandya PK, Olson JC, Oropeza-Vail M, Rai T, Deshpande A. Emergency department visits related to cirrhosis: a retrospective study of the nationwide emergency department sample 2006 to 2011. Medicine (Baltimore). 2015;94(1):e308. http://dx.doi.org/10.1097/MD.0000000000000308

5. Merli M, Lucidi C, Giannelli V, Giusto M, Riggio O, Falcone M, Ridola L, Attili AF, Venditti M. Cirrhotic patients are at risk for health care-associated bacterial infections. Clin Gastroenterol Hepatol. 2010;8(11):979-85. http://dx.doi.org/10.1016/j.cgh.2010.06.024

6. DeRosa FG, Corcione S, Filippini C, Raviolo S, Fossati L, Montrucchio C, Aldieri C, Petrolo A, Cavallo R, Di Perri $\mathrm{G}$. The Effect on mortality of fluconazole or echinocandins treatment in candidemia in internal medicine wards [corrected]. PLoS One. 2015;10(5):e0125149. http://dx.doi.org/10.1371/journal.pone.0125149 
7. Arvaniti V, D’Amico G, Fede G, Manousou P,Tsochatzis E, Pleguezuelo M, Burroughs AK. Infections in patients with cirrhosis increase mortality fourfold and should be used in determining prognosis. Gastroenterology. 2010;139(4):1246-56, 1256.e1-5. http://dx.doi.org/10.1053/j.gastro.2010.06.019

8. Leber B, Spindelboeck W, Stadlbauer V. Infectious complications of acute and chronic liver disease. Semin Respir Crit Care Med. 2012;33(1):80-95. http://dx.doi.org/10.1055/s-0032-1301737

9. Thulstrup AM, Sorensen HT, Schonheyder HC, Moller JK, Tage-Jensen U. Population-based study of the risk and short-term prognosis for bacteremia in patients with liver cirrhosis. Clin Infect Dis. 2000;31(6):1357-61. http://dx.doi.org/10.1086/317494

10. Aggarwal A, Ong JP, Younossi ZM, Nelson DR, HoffmanHogg L, Arroliga AC. Predictors of mortality and resource utilization in cirrhotic patients admitted to the medical ICU. Chest. 2001;119(5):1489-97. http://dx.doi.org/10.1378/chest.119.5.1489

11. Jalan R, Fernandez J, Wiest R, Schnabl B, Moreau R, Angeli P, Stadlbauer V, Gustot T, Bernardi M, Canton R, Albillos A, Lammert F, Wilmer A, Mookerjee R, Vila J, GarciaMartinez R, Wendon J, Such J, Cordoba J, Sanyal A, GarciaTsao G, Arroyo V, Burroughs A, Ginès P. Bacterial infections in cirrhosis: a position statement based on the EASL Special Conference 2013. J Hepatol. 2014;60(6):1310-24. http://dx.doi.org/10.1016/j.jhep.2014.01.024

12. Al-Hasan MN, Lahr BD, Eckel-Passow JE, Baddour LM. Predictive scoring model of mortality in Gramnegative bloodstream infection. Clin Microbiol Infect. 2013;19(10):948-54. http://dx.doi.org/10.1111/14690691.12085

13. De Rosa FG, Corcione S, Raviolo S, Montrucchio C, Aldieri C, Pagani N, Di Perri G. Candidemia, and infections by Clostridium difficile and carbapenemase-producing Enterobacteriaceae: new enteropathogenetic opportunistic syndromes? Infez Med. 2015;23(2):105-16.

14. Kang CI, Song JH, Chung DR, Peck KR, Yeom JS, Ki HK, Son JS, Lee JS, Kim YS, Jung SI, Kim SW, Chang HH, Ryu SY, Kwon KT, Lee H, Jung DS, Moon C, Heo ST, Kim ES, Rhee JY; Korean Network for Study on Infectious Diseases. Liver cirrhosis as a risk factor for mortality in a national cohort of patients with bacteremia. J Infect. 2011;63(5):336-43. http://dx.doi.org/10.1016/j.jinf.2011.07.012

15. Marciano S, Dirchwolf M, Bermudez CS, Sobenko N, Haddad L, Genre F, Barcán L, Smud A, Posadas-Martínez ML, Giunta D, Gadano A. Spontaneous bacteremia and spontaneous bacterial peritonitis share similar prognosis in patients with cirrhosis: a cohort study. Hepatol Int. 2018;12(2):181-190. http://dx.doi.org/10.1007/s12072-017-9837-7

16. European Association for the Study of the Liver. EASL clinical practice guidelines on the management of ascites, spontaneous bacterial peritonitis, and hepatorenal syn- drome in cirrhosis. J Hepatol. 2010;53(3):397-417. http://dx.doi.org/10.1016/j.jhep.2010.05.004

17. Friedman ND, Kaye KS, Stout JE, McGarry SA, Trivette SL, Briggs JP, Lamm W, Clark C, MacFarquhar J, Walton AL, Reller LB, Sexton DJ. Health care--associated bloodstream infections in adults: a reason to change the accepted definition of community-acquired infections. Ann Intern Med. 2002;137(10):791-7. http://dx.doi.org/10.7326/0003-4819-137-10200211190-00007

18. Magiorakos AP, Srinivasan A, Carey RB, Carmeli Y, Falagas ME, Giske CG, Harbarth S, Hindler JF, Kahlmeter G, Olsson-Liljequist B, Paterson DL, Rice LB, Stelling J, Struelens MJ, Vatopoulos A, Weber JT, Monnet DL. Multidrug-resistant, extensively drug-resistant and pandrug-resistant bacteria: an international expert proposal for interim standard definitions for acquired resistance. Clin Microbiol Infect. 2012;18(3):268-81. http://dx.doi.org/10.1111/j.1469-0691.2011.03570.x

19. von Elm E, Altman DG, Egger M, Pocock SJ, Gøtzsche PC, Vandenbroucke JP; STROBE Initiative. The Strengthening the Reporting of Observational Studies in Epidemiology (STROBE) statement: guidelines for reporting observational studies. Ann Intern Med. 2007;147(8):573-7. http://dx.doi.org/10.7326/0003-4819-147-8-20071016000010

20. Campillo B, Dupeyron C, Richardet JP. Epidemiology of hospital-acquired infections in cirrhotic patients: effect of carriage of methicillin-resistant Staphylococcus aureus and influence of previous antibiotic therapy and norfloxacin prophylaxis. Epidemiol Infect. $2001 \mathrm{Dec} ; 127(3): 443-50$. http://dx.doi.org/10.1017/s0950268801006288

21. Munita JM, Araos R, Pérez J, Álvarez A, Canals M, Conteras J, Marcotti A, Thompson L, Noriega LM. Bacteriemia en daño hepático crónico. Rev. chil. infectol. 2011;28(1):35-9. http://dx.doi.org/10.4067/S0716-10182011000100006

22. Albillos A, Lario M, Alvarez-Mon M. Cirrhosis-associated immune dysfunction: distinctive features and clinical relevance. J Hepatol 2014;61(6):1385-96. http://dx.doi.org/10.1016/j.jhep.2014.08.010

23. Bartoletti M, Giannella M, Caraceni P, Domenicali M, Ambretti S, Tedeschi S, Verucchi G, Badia L, Lewis RE, Bernardi M, Viale P. Epidemiology and outcomes of bloodstream infection in patients with cirrhosis. J Hepatol. 2014;61(1):51-8. http://dx.doi.org/10.1016/j.jhep.2014.03.021

24. Hsieh CC, Lee CC, Chan TY, Hong MY, Chi CH, Ko WC. Clinical features and impact of empirical therapy in cirrhotic adults with community-onset bacteremia. Am J Emergency Med. 2015;33(2):222-8. http://dx.doi.org/10.1016/j.ajem.2014.11.024

25. Park H, Jang KJ, Jang W, Park SH, Park JY, Jeon TJ, Oh TH, Shin WC, Choi WC, Sinn DH. Appropriate empirical antibiotic use and 30-d mortality in cirrhotic patients with 
bacteremia. World J Gastroenterol. 2015;21(12):3587-92.

http://dx.doi.org/10.3748/wjg.v21.i12.3587

26. Brandolini M, Corbella M, De Silvestri A, Tinelli C, Albonico G, Albertini R, Ludovisi S, Bruno R, Marone P,
Minoli L, Seminari E. Epidemiological characteristics of bloodstream infections in patients with different degrees of liver disease. Infection. 2015;43(5):561-7. http://dx.doi.org/10.1007/s15010-015-0794-6 